\title{
Copy-Paste Commands with the Additional Facilities
}

\author{
Divij H. Patel \\ Computer Engineering \\ IIET, Dharmaj
}

\begin{abstract}
In present, computer software becomes very well developed. At the starting stage of revolution there were many computer software developed. Best one was operating system which has been necessary for any computer device to perform different operation. They gave important facility to deal with the bytes of data which would be stored on disk or in the document and simply known as copy-paste/cut-paste type operations. But the main problem with this type of operation is that user cannot able to make copy operation again without pasting older one. But present system will provide the facility to make copy operation again and again without past older one and also store the content on the physical memory.
\end{abstract}

\section{Keywords}

Clipboard, copy, paste, editing, real time, handler

\section{INTRODUCTION}

Computing is addition of multiple useful processes and these processes has been upgraded from when the first computer was introduced.

Some of them are cut, copy and paste operation. These three are most important processes but not much as popular so everyone uses it but no one takes too much interest to develop it. People are trying to upgrade the popular processes or softwares. It's a subject to think how these are important. Copy, cut and paste are the basic commands of command prompt and terminal and this entire process handle by the utility called clipboard.

Documentation is being very important part nowadays, everyone from the student to business person need to work on digital documentation and copy the same content from same document to another or from one place to another is also important operation. It's just matter of think that if Cut, paste and copy utility have not been founded then how everyone can transfer the content from one to another place. It's very difficult to transfer text content manually from same document to another document and also it takes to much effort and time. So it's very useful service and also needs to be upgraded as other software systems. Also multiple softwares are available in the market which can store result of multiple copy commands for future use.

But they can't provide real time execution facility and also real time editing of content. In normal, it's necessary to paste the copied content if user wants to copy again otherwise it would be overwrite. In this present process or software, user can store multiple copy results without paste previous one and also can edit it in real time. So it's very useful in documentation.

In the next topic of flow charts are going to discuss how it works by processing flows.

\section{FLOW CHARTS}

In above introduction, paper reflects that what the basic computer commands are like copy, paste and cut operations and how it is useful for documentation. Also got reason why its need to be upgrade by new techniques.

In here present software will be able to provide real time experience and also user can use it by pressing normal hot keys like ctrl+c, ctrl+v and many more.

It can store the every content as a backup, whenever user wants to copy and at the paste time it will show all the content distributed by integer numbers and also with the editing option. Now next is about representation of all the process by flow charts.

Here mainly 4 type of process will take place like:-
1. Set clipboard
2. Get clipboard
3. Store the clipboard value to file
4. Edit content
a. Bold
b. Uppercase
c. Lowercase
d. Translate
e. String operations

First of all content will be store in the clipboard then after it will store as a backup to the temporary file, then after when paste operation will call it will edit the content as per user's choice. Then after it will be paste to the location.

So below flow charts shows the step by step process of present software. 


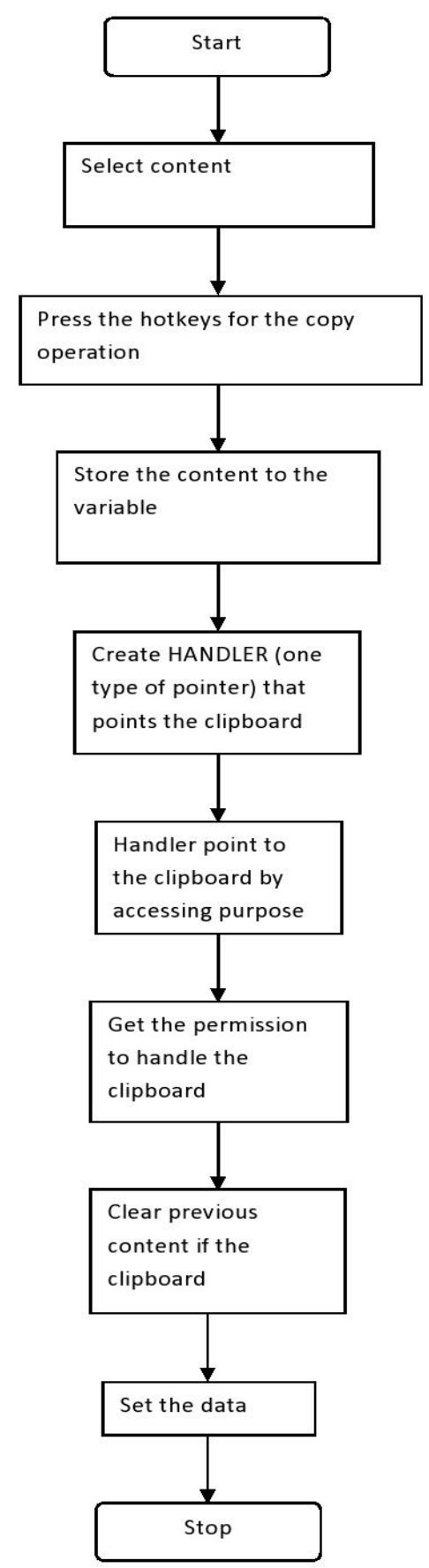

Figure 1: Set the value to the clipboard

After starting the software or process for multiple time copypaste operation user has to select the content and then need to press hot keys for copy like: - ctrl+c. this is the normal steps which has been used every time when ever user wants to copy the content but this flow chart shows the process that how my software manually copy the content or set the clipboard.

After that software variable store the content which has to copy. Then after program will create handler which will be useful to access the clipboard and handler is nothing it's just a one type of pointer then in next step handler will get the permission to access the clipboard.
Then after it will clear previous content if the clipboard if it's there otherwise it will set the new content from the variable. So above mentioned first flow chart shows the process to set the clipboard.

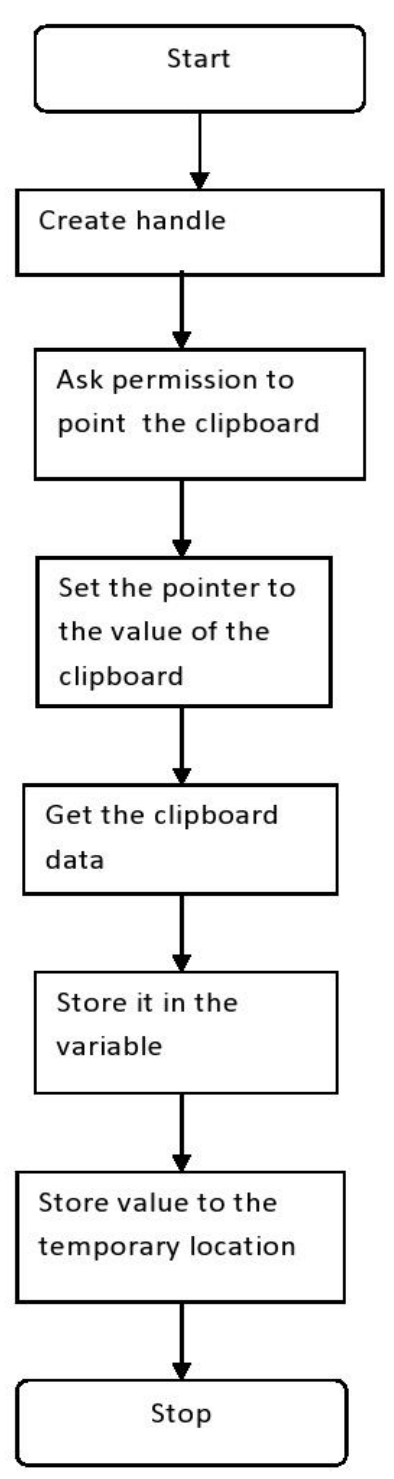

Figure 2: Store the clipboard value to the file

Above first flow chart it shows how to set the clipboard and now in second flow chart it will reflect the information about how to store the content of the clipboard to the temporary location or temporary file.

After setting the clipboard, software handler again ask for the permission to handle the clipboard and then after it will get the clipboard data by "Getclipboard" command. Then after data will be store in the variable and from that variable it will be fetch to the temporary file or path by system file management. 


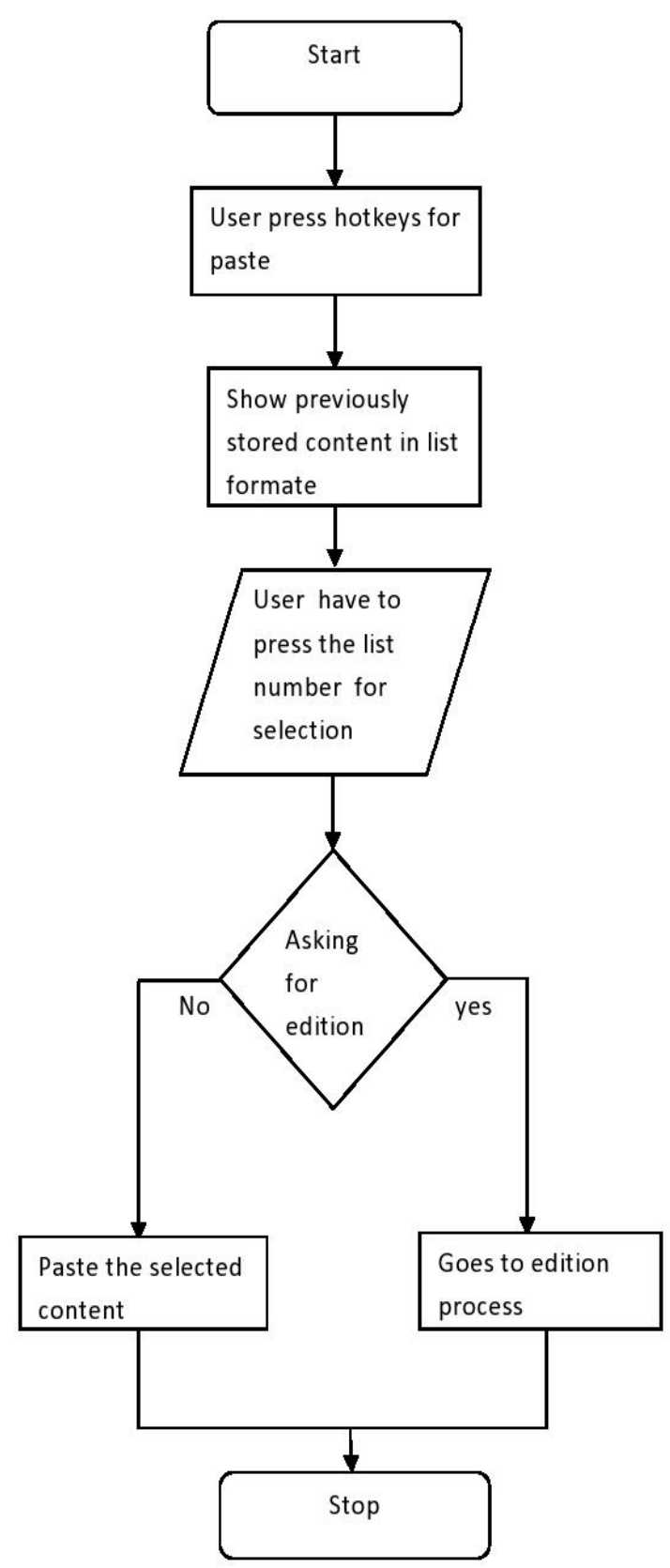

Figure 3: Paste from the list of previous contents

From above mentioned flow chart 1 and flow chart 2 it shows how software manually set the clipboard and gets the data from clipboard to temporary file. Now in this third flow chart, it shows that what will happen at the paste time. First of all when user will press the hotkeys for the paste operation like:ctrl+v, and program will get trigger.

Then it will show the previously stored content from the temporary file. This content will nothing rather than previously copied content and user can use any of this by selecting labeled integer number.

Content that will be shown is in list format and particular integer number given to the every new content, so it becomes easy to choose the proper content.

For example:-
1. First Store content

2. Second store content

3. Third stored content

4. Fourth stored content

5. Fifth stored content

As above example if user wants to paste second stored content then he/she needs to press the integer 2, if user wants to select first stored content then he/she needs to press the integer 1 , same as if user wants to paste the third stored content then he/she needs to press the integer 3 .

This is real time application so that user doesn't need to work with any type of user interface, user just has to press the keyboard key of particular integer number and input will capture by ascii number of pressed key.

After selecting integer number or program will ask for editing option, that user wants to edit the selected text content or not. if user wants to edit it then it will start editing process otherwise same content will be paste.

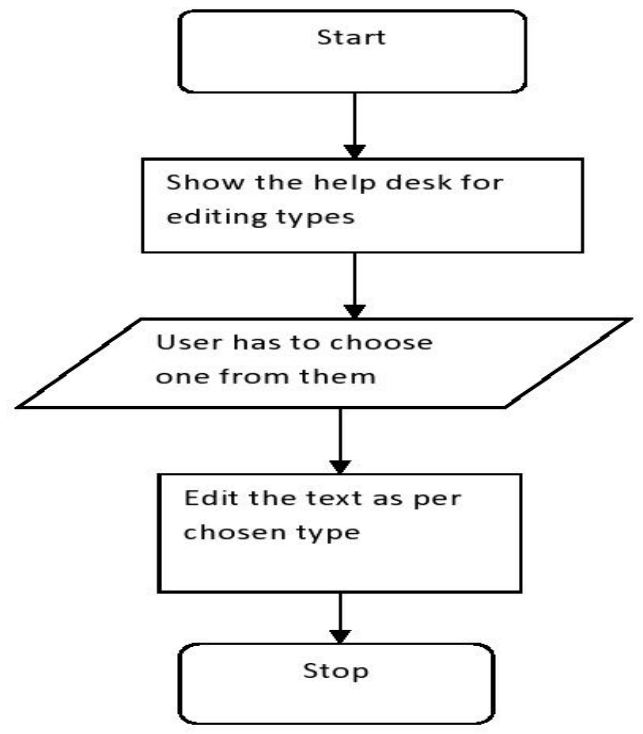

Figure 4: editing

As flow chart 3 shows how paste action will take place but when user will select editing option then help desk will appears.

Help desk shows the options that which type of editing use wants to do with the content. It's like:-

B. bold

U. Upper case

L. Lower case

I. Italic

S. String related operation.

User have to select one appropriate option by just pressing its initial and program will convert it into integer number and capture it as same as previous process. After selecting its content will be store into variable of program and then program will edit it as per user's choice (advance string operations). Then after it will be paste by the "getclipbard" method. 


\section{BENEFITS OF PRESENT PROCESS}

As per above flow charts it's clearly shown the process how it works and now it's time to discuss some benefits of present system over the older one.

Normally copy, paste and cut system only able to paste the copied content, but only once. I mean if user wants to copy next content, then previously copied content automatically removed from the clipboard.

So it's very beneficial to have system that provides facility of paste previously copied contents. Also provide editing option means that when user call for paste command it will show a display with some hot-keys. Like a 'B' for bold, ' $U$ ' for uppercase, 'L' for lower case and many more. So after when user press any hot-key related to selected content and will be edited. Then after it will be paste on a particular location.

It makes our daily work very easy and gives a better user experience. It also use to back - up any content on a particular location only by pressing hot-keys used for copy operation like "ctrl+c". After when user needs it he/she can get it only by pressing hot- keys like "ctrl+v". it also useful with the portable devices like user can locate temporary location to the peripheral device and store whole copied content to the location and user can easily carry the back-up data with the peripheral device.

Main benefit to use this system is that it also works with the device in which it installed. For example after store as a backup in peripheral device if user wants to use it in another device in which the present system is already installed. Then user have to connect peripheral device to the system and locate the temporary storage path then after will be able to used it as a pre-copied data and easily can paste and edit it. It simply shows that copied content of one device also can able to get access in another device, that's very reliable to the user and provide better experience.

\section{EXECUTION}

From above discussion it's clearly understand that how present system will work and what are the benefits of it. Now let's see how practically it works by some screenshots.

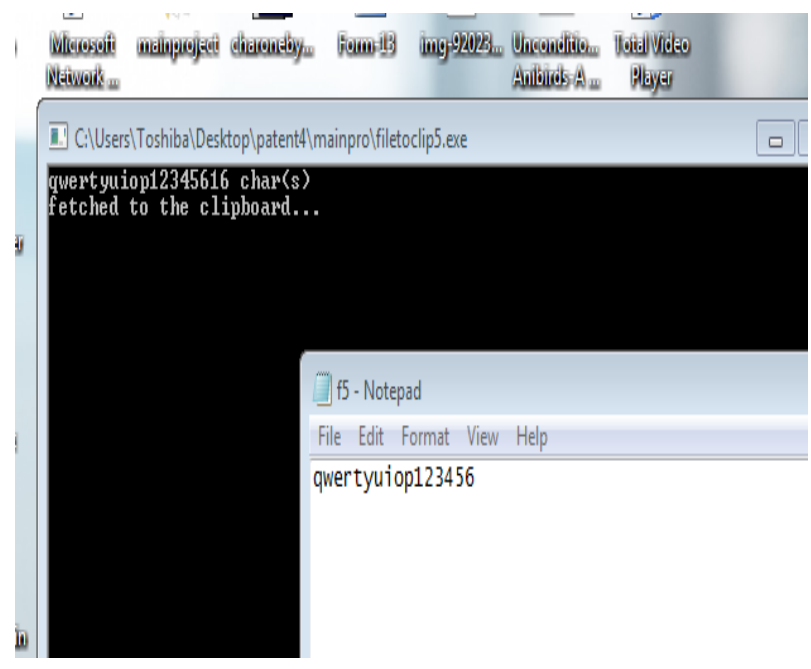

Figure 5: set clipboard

Above figure shows how present software set the clipboard or how it will fetch the content from the file to clipboard. Here in the figure shows that text file having content "qwertyuiop123456" and when user call for the paste operation program fetch that value from temporary file and prompt window shows the output with fetched statement. Temporary file is one type of encrypted file so no one can use it for bad purpose.

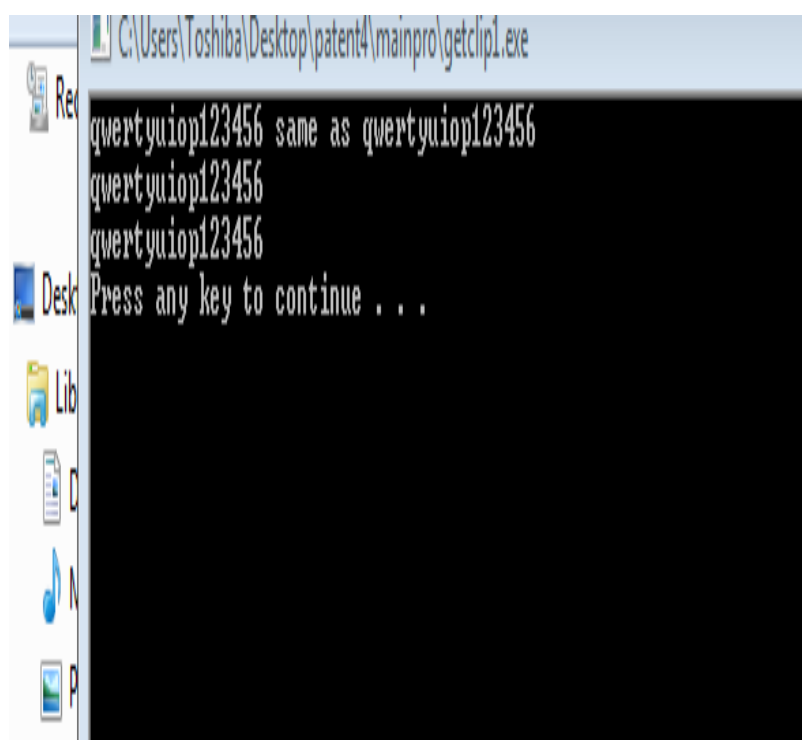

Figure 6: get clipboard

Above figure-6 shows that when user will select editing option before paste the content then how program will fetch the content from clipboard to the variable. In the figure prompt shows the clipboard value that have fetched by the program.

\section{FUTURE SCOPE}

The present software provides facility of multiple time copy operation and additional features like: - editing option and real time input/output services. But in future many other features are going to add as an updated version of present system which will provide translator facility as a editing option. User will be able to translate the content to any language before paste to destination and will also increase interactivity of the system.

\section{CONCLUSION}

Today, Computer technologies being well developed and also with the similar speed software systems reach to the next level like string manipulation, ciphers and many more. So that present system is an updated technique of transferring the content from one to another destination. It provides advance copy, paste operations with the additional features like clipboard editing and real-time input/output. Also it can store the copied content as a backup for the future use and usable in other systems without formatting. Also it will be work as a real time word translation system.

\section{REFERENCES}

[1] Miryung Kim, L. Bergman, T. Lau and D. Notkin, "An ethnographic study of copy and paste programming practices in OOPL, "Empirical Software Engineering, 2004. ISESE '04. Proceedings. 2004 International Symposium on, 2004, pp. 83-92.

[2] US6944821 (B1) - Copy/paste mechanism and paste buffer that includes source information for copied data.

[3] US6240430 (B1) - Method of multiple text selection and manipulation.

[4] US2006218492 (A1) - Copy and paste with citation attributes. 
[5] Sarang B. Kimmatkar, "Collecting clipped data from default clipboard in Linux", Innovations in Information Embedded and Communication Systems (ICIIECS) 2015 International Conference on, pp. 1-4, 2015.

[6] Blaine A. Bell, Steven K. Feiner, Dynamic space management for user interfaces, Proceedings of the 13th annual ACM symposium on User interface software and technology, p.239-248, November 06-08, 2000, San Diego, California, USA.
[7] Francisco Javier Caminero Gil , Fabio Paternò , Vivian Genaro Motti, Context-aware service front-ends, Proceedings of the 5th ACM SIGCHI symposium on Engineering interactive computing systems, June 24-27, 2013, London, United Kingdom.

[8] Z. Li, S. Lu, S. Myagmar, Y. Zhou, "CP-Miner: A Tool for Finding Copy-Paste and Related Bugs in Operating System Code", Proc. Symp. Operating System Design and Implementation, pp. 289-302, 2004. 